\title{
Prevenir e intervenir en los riesgos asociados a las tecnologías de la información y la comunicación: el caso del cyberbullying
}

\author{
Mónica Ojeda Pérez \\ Departamento de Psicología Evolutiva y de la Educación de la Universidad de Sevilla (España) \\ monicaojeda@us.es | https://orcid.org/0000-0001-6056-8595
}

Rosario Del Rey Alamillo

Departamento de Psicología Evolutiva y de la Educación de la Universidad de Sevilla (España) delrey@us.es | https://orcid.org/0000-0002-1907-5489

Este trabajo ha sido seleccionado para su publicación por: don Manuel Area Moreira, doña Eva María Bailén Fernández, don Julio Cabero Almenara, doña Ana García-Valcárcel Muñoz-Repiso, don Pedro José González Felipe y don Alfonso Gutiérrez Martín.

\section{Extracto}

El contexto digital y las tecnologías de la información y la comunicación (TIC) avanzan a una gran velocidad y, aunque presentan múltiples beneficios, sus posibles riesgos también repercuten en la convivencia de los centros educativos. Entre ellos, el cyberbullying es un fenómeno de gran interés, pero sigue siendo necesario comprobar la eficacia de intervenciones basadas en la evidencia para prevenirlo. Por ello, el presente estudio se propuso analizar si es posible disminuir el cyberbullying con una intervención psicoeducativa implementada por el propio profesorado, teniendo en cuenta las diferencias según el género, el curso y los roles de implicación. La muestra estuvo formada por 4.575 estudiantes (48,50\% chicas; $12-16$ años). Los resultados muestran que, con escaso coste y sin requerir una gran especialización, es posible que los centros educativos realicen una intervención psicoeducativa eficaz para la disminución tanto de la «ciberagresión» como de la «cibervictimización». Se proponen estrategias y orientaciones eficaces para el desarrollo de intervenciones psicoeducativas basadas en la evidencia.

Palabras clave: tecnologías de la información y la comunicación (TIC); cyberbullying; adolescencia; prevención; intervención.

Fecha de entrada: 01-06-2020 / Fecha de aceptación: 18-09-2020

Cómo citar: Ojeda Pérez, M. y Del Rey Alamillo, R. (2021). Prevenir e intervenir en los riesgos asociados a las tecnologías de la información y la comunicación: el caso del cyberbullying. Tecnología, Ciencia y Educación, 19, 53-80. https://doi.org/10.51302/tce.2021.612 


\title{
Preventing and intervening in risks associated with information and communication technologies: the case of cyberbullying
}

\author{
Mónica Ojeda Pérez \\ Rosario Del Rey Alamillo
}

Abstract

The digital context and information and communication technologies (ICT) are advancing at a great speed and, although they present multiple benefits, their possible risks also have an impact on the coexistence in schools. Among them, cyberbullying is a phenomenon of great interest, but it is still necessary to verify the effectiveness of evidence-based interventions to prevent it. Therefore, this study aimed to analyse whether it is possible to reduce cyberbullying with a psychoeducational intervention implemented by teachers on their own, considering the differences according to gender, grade and roles of involvement. The sample was formed by 4,575 students ( $48.50 \%$ girls; $12-16$ years old). The results show that, with little cost and without requiring a great deal of specialization, it is possible for schools to carry out an effective psycho-educational intervention to reduce both cyber-perpetration and cyber-victimisation. Effective strategies and guidelines for the development of evidence-based psychoeducational interventions are proposed.

Keywords: information and communications technologies (ICT); cyberbullying; adolescence; prevention; intervention.

Citation: Ojeda Pérez, M. y Del Rey Alamillo, R. (2021). Preventing and intervening in the risks associated with information and communication technologies: the case of cyberbullying. Tecnologia, Ciencia y Educación, 19, 53-80. https://doi.org/10.51302/tce.2021.612 


\section{Sumario}

1. Introducción

1.1. Las relaciones sociales entre iguales a través de las TIC como reto educativo

1.2. Cyberbullying: qué es y por qué prevenirlo

1.3. ¿Se puede prevenir el cyberbullying desde los centros educativos?

2. Objetivos del trabajo

3. Metodología

3.1. Diseño de una intervención psicoeducativa

3.2. Participantes

3.3. Procedimiento

3.4. Instrumento

3.5. Análisis de datos

4. Resultados

4.1. Impacto de la intervención psicoeducativa en el cyberbullying

4.2. Diferencias del impacto de la intervención psicoeducativa en función del género, el curso y los roles del alumnado implicado

5. Discusión

Referencias bibliográficas 


\section{Introducción}

La etapa educativa es esencial en el desarrollo de cualquier adolescente. Durante este periodo deben aprender e integrar nuevos conocimientos y valores que serán clave en su futuro, pero también deben afrontar retos asociados a las relaciones sociales con sus iguales. En este sentido, el Informe Delors (1996) constituyó un punto de inflexión en la concepción de la educación y sus principales pilares -aprender a conocer, aprender a hacer, aprender a ser y aprender a vivir juntos-, fomentando también el trabajo de una convivencia positiva desde los centros educativos. De hecho, la calidad y eficacia educativa ya no solo se mide en función de los resultados finales logrados en cuanto al conocimiento teórico, sino que también deben ser tenidos en cuenta otro tipo de resultados, entre los que la enseñanza orientada a aprender a vivir con los demás ha adquirido una gran importancia (Calvo y Marrero, 2004). Además, durante los últimos años se ha sumado el nuevo contexto de desarrollo posibilitado por las TIC, especialmente las redes sociales virtuales, por el que ya no solo es necesario abordar la convivencia, sino también la ciberconvivencia (Ortega-Ruiz et al., 2012).

La enseñanza orientada a aprender a vivir con los demás ha adquirido una gran importancia. Durante los últimos años ya no solo es necesario abordar la convivencia, sino también la ciberconvivencia

\subsection{Las relaciones sociales entre iguales a través de las TIC como reto educativo}

El estudio de la convivencia escolar ha recibido una gran atención social y científica por su relación con el bienestar y el desarrollo positivo de la comunidad educativa (Del Rey et al., 2017). Se ha convertido en un factor de calidad de la educación. Sin embargo, en los últimos años, el uso de los dispositivos electrónicos y su repercusión en la vida de las personas ha hecho más complejo el entramado de relaciones sociales que constituye la convivencia escolar.

La sociedad actual convive en estrecha relación con las TIC. En la última década, el contexto virtual se ha convertido en un ámbito más de la vida de las personas y, particularmente, de los más jóvenes (Fernández-Montalvo et al., 2015). Los menores pasan más tiempo en internet como
Los menores pasan más tiempo en internet como parte de su cotidianidad y cada vez lo hacen a edades más tempranas


parte de su cotidianidad y cada vez a edades más tempranas, pero es la población adolescente la que parece acceder a internet con mayor frecuencia, desde gran variedad de lugares, y con más flexibilidad y autonomía (Garmendia et al., 2016). Esta relación no solo ha supuesto un antes y un después en cuanto al modo en el que realizan tareas cotidianas, como estudiar o hacer trabajos de clase, sino también en la forma en la que interactúan. Internet y las redes sociales se han configurado como un contexto fundamental donde la población adolescente socializa diariamente (Lenhart et al., 2015).

Tanto es así que la vida social de la población adolescente transcurre, de manera natural, en un continuo donde se unen el entorno online y el offline (Turkle, 2008). Separar hoy en día estos dos contextos sería casi imposible, puesto que se encuentran íntimamente relacionados y, lo que es aún más importante, se retroalimentan. Esto ha dado lugar a un marco de relaciones interpersonales más amplio y complejo que ha aportado nuevos estímulos y formas de comunicación en el día a día (Ortega-Ruiz et al., 2014). Estos cambios se generan, además de en las redes sociales virtuales, en espacios de intercambio de internet y a través de los teléfonos móviles (Bernete, 2010).

Son obvias las ventajas de las comunicaciones digitales, ya que fomentan interacciones positivas y presentan múltiples beneficios, como una mayor facilidad e inmediatez para interactuar. Sin embargo, en función del uso que se haga de estas, también pueden conllevar ciertos riesgos, haciendo más complejo el desarrollo socioemocional de los más jóvenes (RodríguezGómez et al., 2018). Frente a lo que se puede imaginar, estar inmersos en una sociedad digitalizada y pertenecer al grupo de «nativos digitales» no garantiza per se que se cuente con la capacidad crítica y las competencias necesarias para hacer un buen uso de los medios digitales y de las TIC. Es necesaria una alfabetización en medios sociales (Livingstone, 2014). Es por ello por lo que la educación y, concretamente, los centros educativos juegan un papel fundamental también ante esta nueva realidad.

De hecho, ya se ha regulado sobre ello. La reciente Ley orgánica 3/2018, de 5 de diciembre, de protección de datos personales y garantía de los derechos digitales, regula en sus artículos 82, 83 y 84 los derechos a la seguridad digital, la educación digital y la protección de los menores en internet. Concretamente, esta ley señala la necesidad de que las instituciones educativas garanticen el uso seguro y responsable de los medios y dispositivos digitales por parte de los menores, preservando su dignidad y derechos. Sin embargo, va más allá, recomendando también la inclusión de los riesgos que pueden derivar de las TIC como contenidos de las asignaturas escolares, haciendo especial hincapié en las situaciones de violencia. 
Para dar respuesta a esto, los centros educativos han comenzado a incluir la educación sobre el buen uso de las TIC entre sus contenidos. Pero, además, es necesario que el profesorado asuma que las redes sociales virtuales se han establecido como nuevos contextos de socialización que interaccionan con el contexto cara a cara en la escuela. Por tanto, para los centros educativos es fundamental, además, conocer y formar sobre los distintos riesgos que han traspasado sus barreras físicas y afectan también a la convivencia entre su alumnado (véase figura 1). En este sentido, a pesar de ser muchos los tipos de conflictos y episodios de violencia online que pueden influir en las relaciones interpersonales en los centros educativos, el cyberbullying es uno de los fenómenos que más preocupación ha despertado en la sociedad y está suponiendo un reto para los profesionales que trabajan diariamente con adolescentes (Kowalski et al., 2014).

Figura 1. Influencia de las TIC en la convivencia de los centros educativos

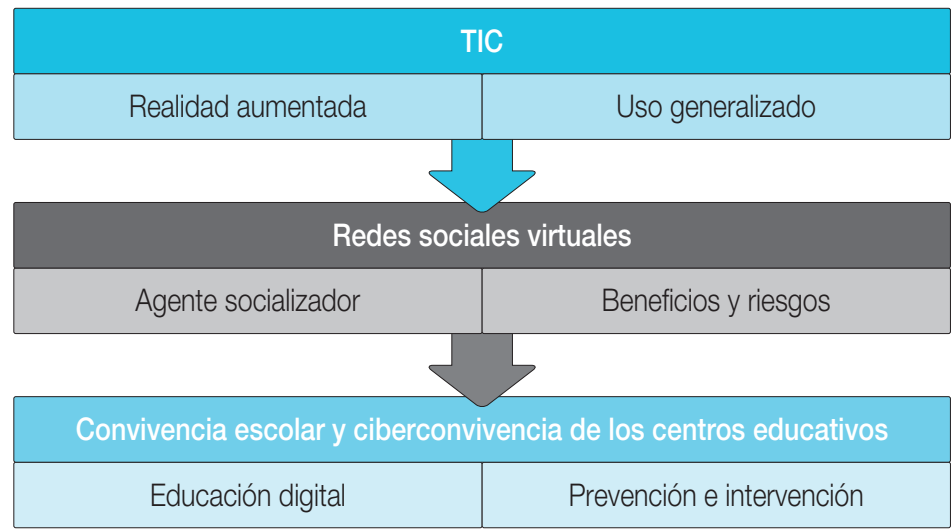

Fuente: elaboración propia

\subsection{Cyberbullying: qué es y por qué prevenirlo}

El cyberbullying es uno de los fenómenos que más ha trascendido a nivel social por su relevancia y sus potenciales consecuencias (Del Rey et al., 2017). En la mayoría de estudios, está siendo analizado teniendo como conocimiento base el bullying, su homólogo en las relaciones 
cara a cara (Garaigordobil, 2015), ya que puede ser entendido como una extensión de este al entorno virtual y a los medios digitales. No obstante, aunque existe un alto grado de coimplicación entre ambos fenómenos (Waasdorp y Bradshaw, 2015), algunas características adquieren significados diferentes debido a la singularidad propia del entorno virtual (Menesini et al., 2012).

El cyberbullying puede definirse como un tipo de agresión injustificada que se produce de manera repetida e intencional entre iguales y se lleva a cabo mediante dispositivos electrónicos (Tokunaga, 2010). De este modo, lo que lo diferencia de agresiones y conflictos puntuales en la red es, al igual que en el caso del bullying, su carácter injustificado, reiterado en el tiempo, intencionado y en el que se produce un desequilibrio de poder por el que la víctima no se siente capaz de defenderse. Sin embargo, algunas características, como la reiteración o el desequilibrio de poder, pueden adquirir matices diferentes en el entorno virtual. Internet facilita una mayor difusión de la agresión, pudiendo llegar a más destinatarios no deseados, aunque el agresor solo lo haya compartido una vez. Asimismo, el desequilibrio de poder puede ser debido a la experiencia y al dominio de las redes sociales e internet, no solo debido a una mayor popularidad o fuerza física. Además, a esto se une la gran cantidad de medios digitales disponibles para agredir y la dificultad que sienten las víctimas para desconectar de las agresiones en cualquier momento, 24 horas al día (Kowalski et al., 2014; Menesini et al., 2012) (véase figura 2).

Figura 2. Conceptualización del cyberbullying

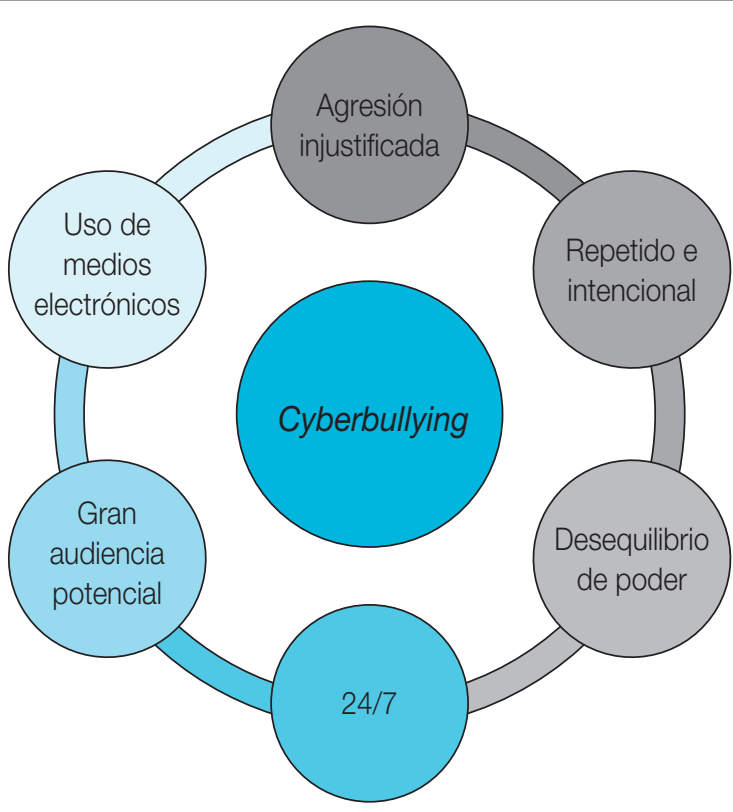

Fuente: elaboración propia 
Este tipo de violencia online está presente en todo el mundo (Kowalski et al., 2014) y, aunque es menos frecuente que el bullying tradicional, parece estar aumentando con el tiempo. En los últimos cinco años, los porcentajes de implicación parece que se han duplicado en todas las edades y niveles socioeconómicos (Garmendia et al., 2016). Concretamente, afecta en torno a 1 de cada 10 menores, siendo el 10,10\% de ellos ciberacosados mediante mensajes y el $8,20 \%$ a través de imágenes (United Nations Educational Scientific and Cultural Organization [UNESCO], 2019). Además, en España, suele pasar por primera vez entre los 8 y los 9 años, y parece que las chicas lo sufren más que los chicos (Sanjuán, 2019; Sastre et al., 2016).

Las consecuencias de este fenómeno pueden ser devastadoras para todas las personas implicadas, con independencia del rol que cada sujeto asuma, ya sea como víctima, agresor o espectador, tanto a corto como a largo plazo. Puede afectar tanto a nivel social y escolar como físico, psicológico y emocional (Fahy et al., 2016; Zych et al., 2015). De hecho, se ha constatado que el cyberbullying puede llegar a alterar el clima escolar del mismo modo que lo hace el bullying tradicional (Tokunaga, 2010) y, además, aumenta el impacto de dichas consecuencias debido a la mayor propagación y alcance que las agresiones pueden tener a través de internet (Zych et al., 2015). Entre otros, puede provocar una mayor frustración, impotencia y falta de seguridad (Hinduja y Patchin, 2008). Además, los comportamientos en línea impactan en la probabilidad de ser acosado online u offline (Seiler y Navarro, 2014). En este sentido, este fenómeno ha mostrado estar relacionado, en muchas ocasiones, con otras prácticas online cuyo mal uso empeora sus posibles consecuencias, como el sexting y el cybergossip (Frankel et al. , 2018; López-Pradas et al., 2017). Por su parte, el sexting hace referencia al intercambio de contenido de carácter erótico o sexual a través de dispositivos y medios electrónicos (Mitchell et al., 2012; Villacampa, 2017). Por otro lado, el cybergossip se refiere a la difusión de rumores a través de internet (Romera et al., 2018). Ambas prácticas se han convertido en nuevas formas mediante las que la población adolescente se puede relacionar online, que también han mostrado estar relacionadas entre sí (Bindesbøl Holm Johansen et al., 2018). En muchas ocasiones, no tienen por qué conllevar consecuencias negativas, pero, en otras, sobre todo cuando se realizan sin consentimiento y para dañar a otras personas, pueden llegar a alcanzar un impacto muy negativo (Rachoene y Oyedemi, 2015; Romera et al., 2018).

Por todo ello, en la sociedad actual, abordar el cyberbullying desde los centros educativos se ha convertido en un aspecto fundamental. Los centros educativos, y, concretamente, el profesorado como perfil mediador, son un contexto clave a través del que prevenir este uso problemático
En la sociedad actual, abordar el gyberbullying desde los centros educativos se ha convertido en un aspecto fundamental 
de las TIC y promover una ciberconvivencia saludable que beneficie un clima escolar positivo. Hoy en día se asume que los planes de convivencia de los centros educativos deben incluir elementos de la ciberconvivencia y casi la totalidad de las comunidades autónomas cuentan con protocolos, de obligado cumplimiento para la comunidad educativa, de detección e intervención ante el cyberbullying, pero no siempre es así (Cerezo y Rubio, 2017).

\section{3. ¿Se puede prevenir el cyberbullying desde los centros edu- cativos?}

La única revisión científica que ha evaluado la efectividad de los programas que abordan el cyberbullying concluyó que no todos son efectivos para prevenir la agresión y victimización del cyberbullying, pero, efectivamente, la prevención de este fenómeno desde los centros educativos sí es posible (Gaffney et al., 2019). No obstante, es necesario comprobar qué intervenciones son eficaces contra el cyberbullying y verificar, además, para qué funcionan y bajo qué circunstancias (Ttofi y Farrington, 2011).

A través de la prevención se pretende evitar, antes de que suceda, un riesgo que provoca daño. Sin embargo, hasta la actualidad, los esfuerzos por regular la actuación ante este fenómeno se han focalizado en el desarrollo de protocolos de actuación sobre cómo se debe intervenir desde los centros educativos cuan-

El gyberbullying sí se puede prevenir desde los centros educativos. No obstante, es necesario comprobar qué intervenciones son eficaces y verificar, además, para qué funcionan y bajo qué circunstancias do ya se ha producido un caso de cyberbullying (por ejemplo, Instrucciones de 11 de enero de 2017). A esto se une, además, que no todas las comunidades españolas contemplan este tipo de violencia online, sino que reducen sus protocolos al bullying tradicional (Rubio et al., 2019). Por ello, además de desarrollar actuaciones de intervención, es necesario incentivar la puesta en práctica de estrategias de prevención.

En este sentido, los centros educativos tienen la opción de incluir en su plan de convivencia escolar actividades destinadas a ayudar a prevenir el cyberbullying y, con este fin, diversas instituciones y asociaciones ofrecen recopilaciones de buenas prácticas. No obstante, en la mayoría de las ocasiones, estas actuaciones responden a la voluntariedad de los propios centros educativos y, para los profesionales de la educación, desarrollar este tipo de actuaciones sin recibir una formación específica sobre este fenómeno y cómo abordarlo puede llegar a ser muy complicado. El cyberbullying ocurre en el entorno virtual, donde algunos docentes se sienten menos cómodos o no se atreven a explorar por su falta de experiencia previa (Patchin e Hinduja, 2012).

Además de ello, aunque multitud de estudios han propuesto estrategias para prevenir el cyberbullying, también se ha destacado la importancia de utilizar prácticas basadas en 
la evidencia científica, es decir, fundamentadas en conocimientos y estrategias que hayan mostrado ser efectivos (Slonje et al., 2013). Por tanto, no solo basta con desarrollar e implementar estrategias de prevención e intervención ante el cyberbullying, sino que también es importante que sean prácticas basadas en la evidencia, que demuestren empíricamente que sirven para lo que dicen servir (Zych et al., 2019).

Es por ello por lo que la formación de los profesionales de la educación que trabajan diariamente con adolescentes, en primer lugar, y la evaluación de las actuaciones que se realizan para actuar ante el cyberbullying, en segundo lugar, son claves para conseguir abordar y prevenir este fenómeno digital de manera eficaz. Para ello, es necesario, por un lado, desarrollar intervenciones basadas en la naturaleza del fenómeno que, además de trabajar con el alumnado, permitan formar previamente al profesorado y, por otro lado, desarrollar estudios longitudinales que comprueben el impacto de estas intervenciones, dónde y cómo funcionan (véase figura 3).

Figura 3. Pautas para la actuación ante el cyberbullying desde los centros educativos

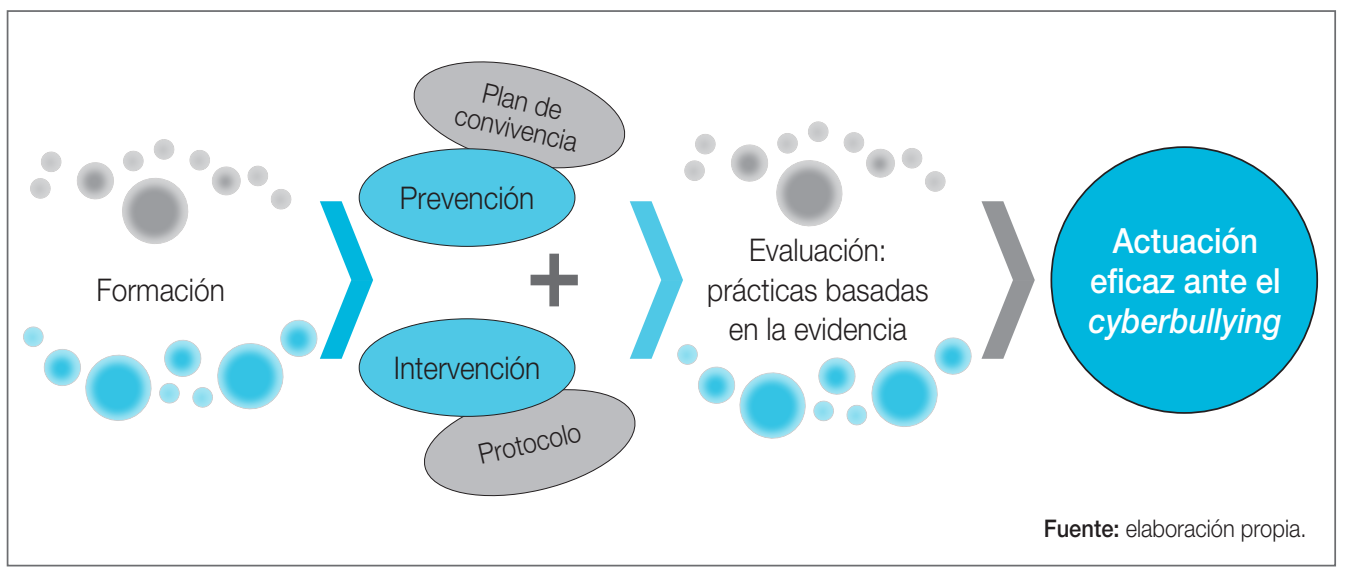

\section{Objetivos del trabajo}

El cyberbullying es actualmente un fenómeno de gran interés para la comunidad científica, la comunidad educativa y, en general, la sociedad en su conjunto. El contexto digital y las TIC avanzan a una gran velocidad y este tipo de violencia online se ha convertido en una forma más mediante la que la población adolescente puede agredir a sus iguales. A pesar 
de la preocupación existente por prevenir este fenómeno, siguen siendo necesarios estudios que permitan comprobar la eficacia de intervenciones psicoeducativas, construidas a partir del conocimiento científico, que aborden el cyberbullying con un bajo coste para los centros educativos. Por ello, tras haber analizado el impacto en un estudio piloto y, posteriormente, haber analizado en una muestra más amplia el impacto en algunas formas de victimización y ciberagresión (Del Rey et al., 2018, 2019), el presente estudio tiene como objetivos:

- Analizar si es posible disminuir el cyberbullying mediante una intervención psicoeducativa implementada por el profesorado.

- Indagar si el impacto de esta intervención psicoeducativa difiere en función del género y el curso del alumnado.

- Examinar en qué roles de implicación del cyberbullying (víctima, agresor, doble rol o no implicados) es posible disminuir la ciberagresión y la cibervictimización del alumnado.

\section{Metodología}

\subsection{Diseño de una intervención psicoeducativa}

La revisión sistemática de la literatura y estudios previos de análisis de la naturaleza del cyberbullying y sus factores asociados (por ejemplo, Álvarez-García et al., 2017; Del Rey et al., 2015; Garaigordobil y Martínez-Valderrey, 2015; OrtegaRuiz et al., 2016; Zych et al., 2015) permitió contar con evidencias científicas suficientes para el diseño de una intervención psicoeducativa para la prevención e intervención del cyberbullying (Del Rey et al., 2018, 2019).

La intervención sienta sus bases en la teoría del comportamiento social normativo (Rimal et al.,

La intervención psicoeducativa sienta sus bases en la teoría del comportamiento social normativo [...]. Además, se basa en las habilidades de autorregulación del alumnado [...], incluyendo actividades reflexivas que fomentan las habilidades metacognitivas y la construcción del conocimiento a partir de las ideas previas 2005). Esta teoría sostiene que la conducta de las personas está influenciada por las normas y creencias sociales que perciben y, por tanto, si se influye sobre las creencias, se puede lograr un cambio en el comportamiento de la población adolescente. Además, se basa en las habilidades de autorregulación del alumnado (Nacimiento et al., 2017), incluyendo actividades reflexivas que fomentan las habilidades metacognitivas y la construcción del conocimiento a partir de las ideas previas (Ausubel, 1963; Powell y Cody, 2009). 
Además de otorgar un papel protagonista al alumnado, se incorpora la participación del profesorado como un elemento clave. Esto facilita que los centros educativos puedan desarrollar la intervención con sus propios medios, adaptándola a su contexto particular y sin depender de agentes externos. Asimismo, se ha demostrado que la percepción que el alumnado tenga sobre su relación con el profesorado es fundamental para el éxito de las actividades que se desarrollen (Espelage y Swearer, 2008). Así, la implicación, las expectativas, la motivación y la actitud del profesorado han mostrado ser determinantes para la efectividad de la intervención psicoeducativa. De hecho, al igual que ocurre en el bullying tradicional, los menores consideran que el grado de implicación del profesorado es un factor muy relevante para aumentar o disminuir su participación (Casas et al., 2015). Es por ello por lo que el propio profesorado del centro educativo, de forma autónoma, con el manual y los materiales audiovisuales facilitados, desarrolla las sesiones con su alumnado.

El material educativo cuenta con recursos de concienciación, como carteles, pegatinas o marcadores, posibilitando el desarrollo de una campaña de sensibilización que dé unidad a la intervención. También, incluye un manual y material audiovisual para ser utilizado con el alumnado y sus familias, puesto que involucrar a estas últimas es también fundamental para dar coherencia a la actuación. El manual es una guía de enseñanza útil para aprender de manera autónoma, que proporciona directrices tanto generales como específicas. No obstante, el profesorado tiene libertad para cambiar recursos y adaptarlos a su situación particular, si así lo considera necesario. Se ofrece una descripción completa de la intervención, su metodología y los pasos que hay que seguir con el alumnado, además de recursos, explicaciones adicionales y orientaciones específicas, con información clara y concisa sobre el cyberbullying. También se incluye un glosario de términos, un banco de recursos (como descripciones de los youtubers más populares) o enlaces a lecturas adicionales. Además, se proporcionan las claves para evaluar cada sesión y la intervención psicoeducativa en su conjunto.

La intervención consta de ocho sesiones para educación secundaria obligatoria (ESO) y aborda el cyberbullying, pero también incluye riesgos asociados a este, como el bullying, el cybergossip, el sexting y el uso abusivo de las redes sociales e internet. Para ello, se utilizan actividades con simulaciones basadas en el entorno online y offline y se trabaja a través del análisis de situaciones cotidianas del propio alumnado y su entorno, incluyendo sus creencias previas y la normalización de los comportamientos online. La metodología utilizada es
El material educativo cuenta con recursos de concienciación, como carteles, pegatinas o marcadores, posibilitando el desarrollo de una campaña de sensibilización que dé unidad a la intervención.
También, incluye un manual y material audiovisual para ser utilizado con el alumnado y sus familias, pues involucrar a estas últimas es fundamental 
una de las características clave de esta intervención. Cada sesión contiene una actividad específica que asegura que se cumplan los requisitos de la metodología, de modo que todas las sesiones están diseñadas con una secuencia similar. Esta secuencia se compone de cinco actividades base que se adaptan a cada sesión (véase figura 4).

Figura 4. Secuencia de actividades de las sesiones

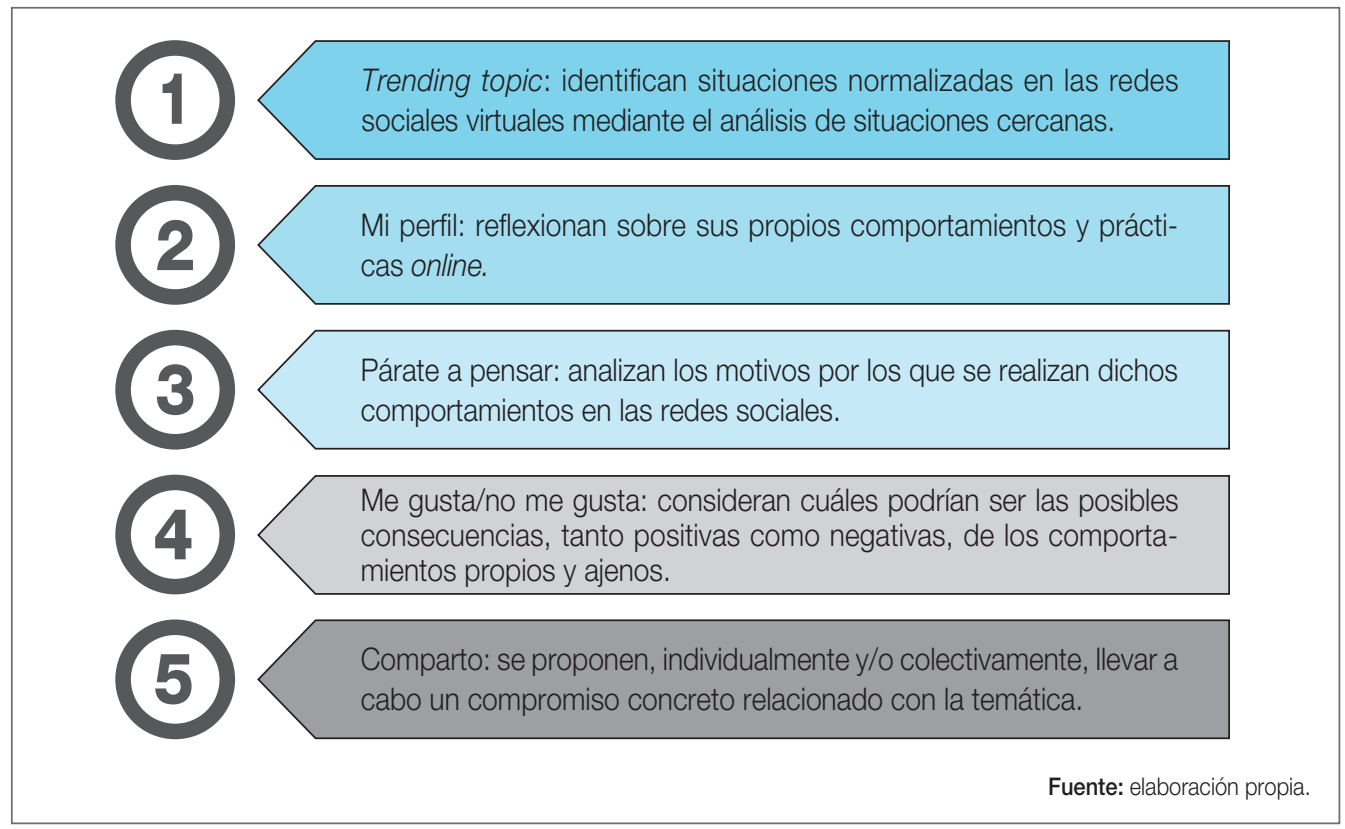

\subsection{Participantes}

El universo de análisis de esta investigación es la población adolescente escolarizada en ESO, dado el uso generalizado de las redes sociales virtuales en esta edad. En total, participaron 4.575 estudiantes (48,50\% chicas) de entre 12 y 16 años $\left(M_{\text {edad }}=13,46\right.$; $\left.\mathrm{DT}_{\text {edad }}=1,19\right)$ procedentes de 19 centros educativos (7 control y 14 cuasiexperimental). La muestra en la primera recogida de datos (T1) está formada por 4.198 estudiantes (48,80 \% chicas) con edades comprendidas entre los 12 y los 16 años $\left(M_{\text {edad }}=13,45 ; D T_{\text {edad }}=1,20\right)$. La muestra en la segunda recogida de datos (T2) está formada por 3.393 estudiantes $(49,40 \%$ chicas) con edades comprendidas entre los 12 y los 17 años
El universo de análisis de esta investigación es la población adolescente escolarizada en ESO, dado el uso generalizado de las redes sociales virtuales en esta edad. Participaron 4.575 estudiantes


$\left(M_{\text {edad }}=13,69 ; D T_{\text {edad }}=1,21\right)$. El cuadro 1 indica las características de la muestra en función del grupo control/cuasiexperimental.

Cuadro 1. Características de la muestra en función de la condición control/cuasiexperimental

\begin{tabular}{|c|c|c|c|c|c|c|c|}
\hline & & \multicolumn{2}{|c|}{ Control } & \multicolumn{2}{|c|}{ Cuasiexperimental } & \multicolumn{2}{|c|}{ Total } \\
\hline \multicolumn{2}{|c|}{ Variables } & $\mathbf{N}$ & $\%$ & $\mathbf{N}$ & $\%$ & $\mathbf{N}$ & $\%$ \\
\hline \multirow{2}{*}{ Género } & Chica & 801 & 47,70 & 1.416 & 49 & 2.217 & 48,50 \\
\hline & Chico & 878 & 52,30 & 1.473 & 51 & 2.351 & 51,50 \\
\hline \multirow{5}{*}{ Edad } & 12 & 389 & 23,10 & 785 & 27,10 & 1.174 & 25,70 \\
\hline & 13 & 500 & 29,70 & 869 & 30 & 1.369 & 29,90 \\
\hline & 14 & 380 & 22,60 & 661 & 22,80 & 1.041 & 22,80 \\
\hline & 15 & 313 & 18,60 & 402 & 13,90 & 715 & 15,60 \\
\hline & 16 & 99 & 5,90 & 177 & 6,10 & 276 & 6 \\
\hline \multirow{4}{*}{ Curso } & $1 .^{\circ}$ de ESO & 580 & 34,50 & 1.271 & 43,90 & 1.851 & 40,50 \\
\hline & 2..$^{\circ}$ de ESO & 504 & 30 & 958 & 33,10 & 1.462 & 32 \\
\hline & 3..$^{\circ}$ de ESO & 346 & 20,60 & 464 & 16 & 810 & 17,70 \\
\hline & 4. ${ }^{\circ}$ de ESO & 251 & 14,90 & 201 & 6,90 & 452 & 9,90 \\
\hline \multirow{9}{*}{$\begin{array}{c}\text { Redes } \\
\text { sociales } \\
\text { utilizadas }\end{array}$} & Ninguna & 24 & 1,60 & 55 & 2,10 & 79 & 1,90 \\
\hline & Al menos una & 1.503 & 98,40 & 2.609 & 97,90 & 4.112 & 98,10 \\
\hline & WhatsApp & 1.480 & 96,90 & 2.554 & 96 & 4.034 & 96,30 \\
\hline & Instagram & 1.227 & 80,40 & 2.102 & 80,30 & 3.329 & 80,30 \\
\hline & Snapchat & 675 & 44,20 & 1.122 & 43,90 & 1.797 & 44 \\
\hline & Facebook & 289 & 18,90 & 795 & 31,50 & 1.084 & 26,80 \\
\hline & Twitter & 389 & 23,10 & 601 & 23,40 & 990 & 24,20 \\
\hline & Telegram & 71 & 4,60 & 103 & 4,20 & 174 & 4,40 \\
\hline & Tinder & 5 & 0,30 & 11 & 0,40 & 16 & 0,30 \\
\hline
\end{tabular}




\subsection{Procedimiento}

Tras el diseño de la intervención, se contactó con los equipos directivos de diversos centros educativos para que participaran en un estudio sobre el uso de las redes sociales y los riesgos asociados a ello y el impacto de una intervención psicoeducativa. El muestreo realizado fue, por tanto, incidental por accesibilidad, pero, con objeto de conseguir la máxima representatividad, se seleccionaron centros de diversas provincias andaluzas y se intentó que en la muestra se encontrase representada toda la diversidad de centros educativos de educación secundaria en cuanto a su adscripción (centros públicos ordinarios, centros públicos de atención preferente y centros concertados).

Tras ello, se incluyeron en el estudio los centros educativos que manifestaron su interés. De manera conjunta, se decidió qué centros iban a ser controles (no implementaban la intervención) y cuáles cuasiexperimentales (sí implementaban la intervención), así como las clases que participarían en el estudio.

Una vez que se recibieron los permisos de cada Consejo Escolar, se procedió a la primera recogida de datos en todos los centros (T1), previa a la implementación de la intervención en los centros cuasiexperimentales. Se administraron cuestionarios en papel y lápiz durante el horario de clase por personas investigadoras y docentes que habían recibido formación previa. Durante la administración de los cuestionarios, se hizo hincapié en la naturaleza anónima y voluntaria de la participación, en el tratamiento confidencial de los datos y en la importancia de la sinceridad en las respuestas.

Tras ello, se entregó de manera gratuita al profesorado el paquete con el material educativo. En los centros educativos cuasiexperimentales, se realizaron campañas de sensibilización y se facilitó una sesión de formación al profesorado y otra a las familias. Posteriormente, el profesorado llevó a cabo las sesiones. Una vez que los centros cuasiexperimentales completaron la intervención, aproximadamente a los cuatro meses, se volvieron a administrar los cuestionarios (T2) en todos los centros educativos, control y cuasiexperimental, siguiendo el mismo procedimiento. A los centros educativos que participaron como grupos control, en los que la intervención no se llevó a cabo, se les ofreció que la desarrollasen una vez que el estudio se hubiese completado.

\subsection{Instrumento}

Se utilizó la escala European cyberbullying intervention project questionnaire (ECIPQ) (Del Rey et al., 2015) para evaluar la frecuencia de implicación en cyberbullying. Esta escala está compuesta por 22 ítems y dos dimensiones: ciberagresión (11 ítems) y cibervictimización (11 ítems). Ambas dimensiones evalúan la implicación en los dos últimos meses y cuentan con cinco opciones de respuesta en formato Likert: 0 = No; 1 = Sí, una o dos veces; 2 = Sí, una o dos veces al mes; 3 = Sí, una o dos veces a la semana; y 4 = Sí, más de una vez a la 
semana. Un ejemplo de ítem de la dimensión de ciberagresión es el siguiente: «He excluido o ignorado a alguien en internet, las redes sociales o grupo de WhatsApp». Un ejemplo de ítem de la dimensión de cibervictimización es el siguiente: «Alguien me ha dicho palabras malsonantes o me ha insultado usando internet, las redes sociales o WhatsApp». La fiabilidad de la escala para el presente estudio es $\alpha=0,81, \alpha=0,76$ para la dimensión de cibervictimización y $\alpha=0,72$ para la dimensión de ciberagresión.

\subsection{Análisis de datos}

La codificación y el análisis de los datos se realizó con el programa SPSS, V26. Con el fin de conseguir los objetivos propuestos, se realizaron análisis descriptivos y pruebas estadísticas básicas. Asimismo, se realizaron 22 anovas de medidas repetidas con dos tiempos (T1 y T2) y dos condiciones (control y cuasiexperimental) para comparar el impacto de la intervención psicoeducativa en la implicación en la ciberagresión y cibervictimización de cyberbullying.

\section{Resultados}

\subsection{Impacto de la intervención psicoeducativa en el cyberbullying}

Los resultados mostraron un impacto significativo de la intervención a nivel intrasujeto del tiempo, tanto en la ciberagresión $\left(F_{(1,1)}=10,27 ; p=, 001\right)$ como en la cibervictimización $\left(F_{(1,1)}=11,43 ; p=, 001\right)$, pero no a nivel intersujeto de la condición, ni en la ciberagresión $\left(F_{(1,1)}=2,27 ; p=, 132\right)$ ni en la cibervictimización $\left(F_{(1,1)}=0,35 ; p=, 552\right)$. Estos datos indican una clara reducción de la ciberagresión y de la cibervictimización asociada a la intervención, tal y como se puede observar al comparar las medias (véase cuadro 2).

Cuadro 2. Medias de cyberbullying en T1 y T2 en función de la condición

\begin{tabular}{l|l|c|c}
\multirow{2}{*}{ Dimensión } & \multicolumn{1}{c}{ Condición } & \multicolumn{2}{c}{ M (DT) } \\
\cline { 3 - 4 } & Control $(n=1.183)$ & T1 & T2 \\
\cline { 2 - 4 } Ciberagresión & Cuasiexperimental $(n=1.666)$ & $0,08(0,16)$ & $0,08(0,20)$ \\
\cline { 2 - 4 } & Control $(n=1.189)$ & $0,10(0,16)$ & $0,07(0,21)$ \\
\hline \multirow{2}{*}{ Cibervictimización } & Cuasiexperimental $(n=1.664)$ & $0,15(0,25)$ & $0,14(0,28)$ \\
\cline { 2 - 4 } & & $0,12(0,25)$ \\
\hline
\end{tabular}




\subsection{Diferencias del impacto de la intervención psicoeducativa en función del género, el curso y los roles del alumnado im- plicado}

Entre las chicas, los resultados mostraron un impacto significativo de la intervención a nivel intrasujeto del tiempo en la ciberagresión $\left(F_{(1,1)}=7,06 ; p=, 008\right)$, pero no en la cibervictimización $\left(F_{(1,1)}=3,47 ; p=\right.$,063). Tampoco se han encontrado diferencias a nivel intersujeto de la condición, ni en la ciberagresión $\left(F_{(1,1)}=1,83 ; p=, 177\right)$ ni en la cibervictimización $\left(F_{(1,1)}=0,06 ; p=, 809\right)$. Estos datos indican una clara reducción de la ciberagresión asociada a la intervención, tal y como se puede observar al comparar las medias (véase cuadro 3).

En los chicos, se ha encontrado un impacto significativo de la intervención a nivel intrasujeto del tiempo, tanto en la ciberagresión $\left(F_{(1,1)}=4,23 ; p=, 040\right)$ como en la cibervictimización $\left(F_{(1,1)}=8,03 ; p=, 005\right)$, pero no a nivel intersujeto de la condición, ni en la ciberagresión $\left(F_{(1,1)}=0,77 ; p=, 379\right)$ ni en la cibervictimización $\left(F_{(1,1)}=0,33 ; p=, 566\right)$. Estos datos indican una clara reducción de la ciberagresión y la cibervictimización asociada a la intervención, tal y como se puede observar al comparar las medias (véase cuadro 3).

Cuadro 3. Medias de cyberbullying en T1 y T2 en función de la condición y el género

\begin{tabular}{|c|c|c|c|}
\hline \multirow{2}{*}{ Dimensión } & \multirow{2}{*}{ Condición } & \multicolumn{2}{|c|}{ M (DT) } \\
\hline & & T1 & $\mathrm{T} 2$ \\
\hline \multicolumn{4}{|c|}{ Chicas } \\
\hline \multirow{2}{*}{ Ciberagresión } & Control $(n=577)$ & $0,08(0,15)$ & $0,07(0,15)$ \\
\hline & Cuasiexperimental $(n=849)$ & $0,10(0,19)$ & $0,07(0,17)$ \\
\hline \multirow{2}{*}{ Cibervictimización } & Control $(n=572)$ & $0,14(0,21)$ & $014(0,28)$ \\
\hline & Cuasiexperimental $(n=842)$ & $0,15(0,26)$ & $0,13(0,25)$ \\
\hline \multicolumn{4}{|c|}{ Chicos } \\
\hline \multirow{2}{*}{ Ciberagresión } & Control $(n=606)$ & $0,08(0,18)$ & $0,09(0,23)$ \\
\hline & Cuasiexperimental $(n=817)$ & $0,10(0,22)$ & $0,08(0,24)$ \\
\hline \multirow{2}{*}{ Cibervictimización } & Control $(n=617)$ & $0,12(0,21)$ & $0,14(0,28)$ \\
\hline & Cuasiexperimental $(n=822)$ & $0,15(0,25)$ & $0,12(0,25)$ \\
\hline
\end{tabular}


En cuanto al curso, en $1 .^{\circ}$ de ESO, los resultados mostraron un impacto significativo de la intervención a nivel intrasujeto del tiempo en la cibervictimización $\left(F_{(1,1)}=10,38 ; p=\right.$ $=, 001)$, pero no en la ciberagresión $\left(F_{(1,1)}=0,11 ; p=, 744\right)$. Tampoco se han encontrado diferencias a nivel intersujeto de la condición, ni en la cibervictimización $\left(F_{(1,1)}=1,24\right.$; $p=, 265)$ ni en la ciberagresión $\left(F_{(1,1)}=0,31 ; p=, 578\right)$. Estos datos indican una clara reducción de la cibervictimización asociada a la intervención, tal y como se puede observar al comparar las medias (véase cuadro 4).

En $2 .^{\circ}$ de ESO se ha encontrado un impacto significativo de la intervención a nivel intrasujeto del tiempo en la ciberagresión $\left(F_{(1,1)}=5,38 ; p=, 021\right)$, pero no en la cibervictimización $\left(F_{(1,1)}=0,89 ; p=, 345\right)$. Tampoco se han encontrado diferencias a nivel intersujeto de la condición, ni en la ciberagresión $\left(F_{(1,1)}=2,62 ; p=, 106\right)$ ni en la cibervictimización $\left(F_{(1,1)}=0,22 ; p=, 638\right)$. Estos datos indican una clara reducción de la ciberagresión asociada a la intervención, tal y como se puede observar al comparar las medias (véase cuadro 4).

En $3 .^{\circ}$ de ESO se ha encontrado un impacto significativo de la intervención a nivel intrasujeto del tiempo en la ciberagresión $\left(F_{(1,1)}=11,71 ; p=, 001\right)$, pero no en la cibervictimización $\left(F_{(1,1)}=0,00 ; p=, 948\right)$. Tampoco se han encontrado diferencias a nivel intersujeto de la condición, ni en la ciberagresión $\left(F_{(1,1)}=1,83 ; p=, 177\right)$ ni en la cibervictimización $\left(F_{(1,1)}=1,51 ; p=, 220\right)$. Estos datos indican una clara reducción de la ciberagresión asociada a la intervención, tal y como se puede observar al comparar las medias (véase cuadro 4).

En $4 .^{\circ}$ de ESO no se ha encontrado un impacto significativo de la intervención a nivel intrasujeto del tiempo, ni en la ciberagresión $\left(F_{(1,1)}=0,08 ; p=, 782\right)$ ni en la cibervictimización $\left(F_{(1,1)}=0,13 ; p=, 717\right)$. Sí se han encontrado diferencias a nivel intersujeto de la condición en la ciberagresión $\left(F_{(1,1)}=4,18 ; p=, 042\right)$, pero no en la cibervictimización $\left(F_{(1,1)}=0,01\right.$; $p=, 926)$. Concretamente, en este curso, el cyberbullying disminuye de manera generalizada en ambos grupos, tanto control como cuasiexperimental, por lo que no existe diferencia entre ambos (véase cuadro 4).

Cuadro 4. Medias de cyberbullying en T1 y T2 en función de la condición y el curso

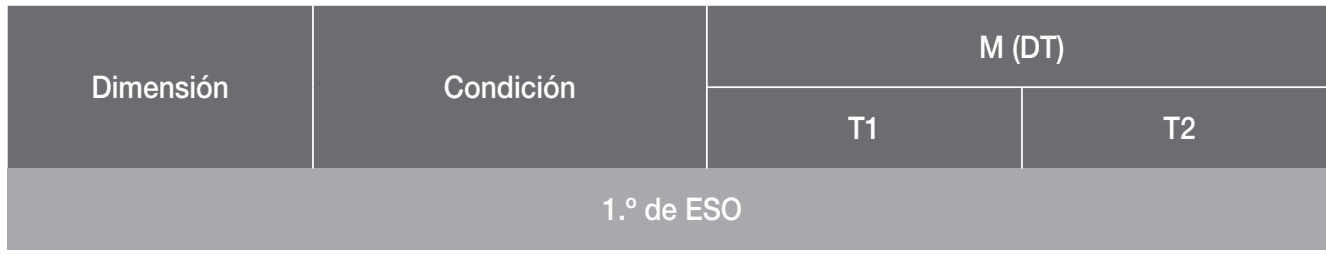

Ciberagresión

$$
\text { Control }(n=388)
$$

$0,08(0,16)$

$0,06(0,17)$

$$
\text { Cuasiexperimental }(n=696)
$$

$0,08(0,20)$

$0,06(0,19)$ 

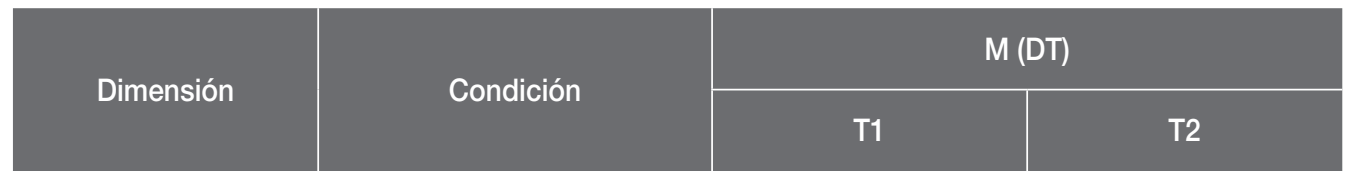
Control $(n=387)$
$0,12(0,19)$
$0,11(0,28)$

Cibervictimización
Cuasiexperimental $(n=702)$
$0,16(0,27)$
$0,11(0,21)$

\section{$2 .^{\circ}$ de ESO}

\begin{tabular}{|c|c|c|c|}
\hline \multirow{2}{*}{ Ciberagresión } & Control $(n=348)$ & $0,07(0,17)$ & $0,07(0,17)$ \\
\hline & Cuasiexperimental $(n=579)$ & $0,11(0,22)$ & $0,07(0,23)$ \\
\hline \multirow{2}{*}{ Cibervictimización } & Control $(n=357)$ & $0,14(0,24)$ & $0,15(0,30)$ \\
\hline & Cuasiexperimental $(n=580)$ & $0,16(0,27)$ & $0,15(0,31)$ \\
\hline \multicolumn{4}{|c|}{$3^{\circ}$ de ESO } \\
\hline \multirow{2}{*}{ Ciberagresión } & Control $(n=243)$ & $0,08(0,16)$ & $0,12(0,28)$ \\
\hline & Cuasiexperimental $(n=283)$ & $0,12(0,20)$ & $0,09(0,21)$ \\
\hline \multirow{2}{*}{ Cibervictimización } & Control $(n=242)$ & $0,13(0,23)$ & $0,16(0,31)$ \\
\hline & Cuasiexperimental $(n=271)$ & $0,13(0,21)$ & $0,12(0,19)$ \\
\hline \multicolumn{4}{|c|}{$4 .^{\circ}$ de ESO } \\
\hline \multirow{2}{*}{ Ciberagresión } & Control $(n=204)$ & $0,08(0,17)$ & $0,06(0,14)$ \\
\hline & Cuasiexperimental $(n=108)$ & $0,12(0,16)$ & $0,09(0,20)$ \\
\hline \multirow{2}{*}{ Cibervictimización } & Control $(n=203)$ & $0,13(0,20)$ & $0,12(0,19)$ \\
\hline & Cuasiexperimental $(n=111)$ & $0,14(0,20)$ & $0,11(0,20)$ \\
\hline
\end{tabular}

Fuente: elaboración propia.

Con respecto a los roles del alumnado implicado, entre las víctimas, los resultados mostraron un impacto significativo de la intervención a nivel intrasujeto del tiempo en la cibervictimización $\left(F_{(1,1)}=7,65 ; p=, 006\right)$, pero no en la ciberagresión $\left(F_{(1,1)}=0,94 ; p=, 334\right)$. 
Tampoco se han encontrado diferencias a nivel intersujeto de la condición, ni en la cibervictimización $\left(F_{(1,1)}=0,08 ; p=, 784\right)$ ni en la ciberagresión $\left(F_{(1,1)}=0,04 ; p=, 852\right)$. Estos datos indican una clara reducción de la cibervictimización asociada a la intervención, tal y como se puede observar al comparar las medias (véase cuadro 5).

Entre los agresores, se ha encontrado un impacto significativo de la intervención a nivel intrasujeto del tiempo en la cibervictimización $\left(F_{(1,1)}=4,97 ; p=, 028\right)$, pero no en la ciberagresión $\left(F_{(1,1)}=0,19 ; p=, 663\right)$. Se han encontrado diferencias a nivel intersujeto de la condición en la cibervictimización $\left(F_{(1,1)}=13,46 ; p=, 000\right)$, pero no en la ciberagresión $\left(F_{(1,1)}=0,24\right.$; $p=$,622). Estos datos indican una clara reducción de la cibervictimización asociada a la intervención, tal y como se puede observar al comparar las medias (véase cuadro 5).

Entre quienes comparten el doble rol (víctima-agresor), no se ha encontrado un impacto significativo de la intervención a nivel intrasujeto del tiempo, ni en la ciberagresión $\left(F_{(1,1)}=2,77 ; p=, 100\right)$ ni en la cibervictimización $\left(F_{(1,1)}=0,35 ; p=, 558\right)$. Tampoco se han encontrado diferencias a nivel intersujeto de la condición, ni en la ciberagresión $\left(F_{(1,1)}=0,20\right.$; $p=, 653)$ ni en la cibervictimización $\left(F_{(1,1)}=0,33 ; p=, 569\right)$. Estos datos indican que este rol es al que menos impacta la intervención (véase cuadro 5).

Entre las personas no implicadas, se ha encontrado un impacto significativo de la intervención a nivel intrasujeto del tiempo en la ciberagresión $\left(F_{(1,1)}=4,19 ; p=, 041\right)$, pero no en la cibervictimización $\left(F_{(1,1)}=2,36 ; p=, 124\right)$. Tampoco se han encontrado diferencias a nivel intersujeto de la condición, ni en la ciberagresión $\left(F_{(1,1)}=0,63 ; p=, 428\right)$ ni en la cibervictimización $\left(F_{(1,1)}=1,40 ; p=, 237\right)$. Estos datos indican una clara reducción de la ciberagresión asociada a la intervención, tal y como se puede observar al comparar las medias (véase cuadro 5).

Cuadro 5. Medias de cyberbullying en T1 y T2 en función de la condición y el rol

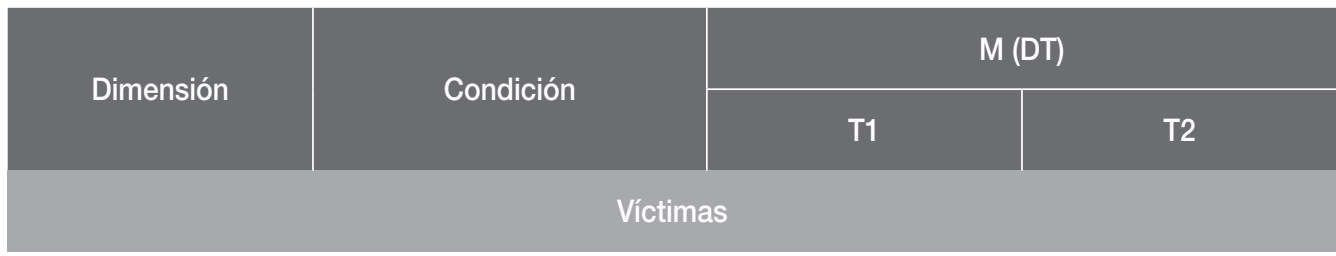

Ciberagresión

Control $(n=95)$

$0,10(0,13)$

$0,12(0,27)$

Cuasiexperimental $(n=124)$

$0,11(0,13)$

$0,10(0,21)$

Cibervictimización

$$
\text { Control }(n=98)
$$

$0,53(0,26)$

$0,41(0,57)$

Cuasiexperimental $(n=131)$

$0,63(0,42)$

$0,34(0,45)$ 

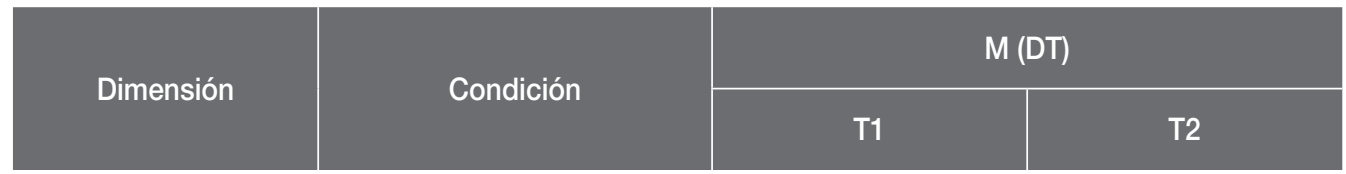

\section{Agresores}

$$
\text { Control }(n=40)
$$

$$
0,49(0,27)
$$

$0,24(0,24)$

Ciberagresión
Cuasiexperimental $(n=73)$
$0,53(0,32)$
$0,25(0,34)$
Control $(n=42)$
$0,23(0,17)$
$0,32(0,28)$

Cibervictimización
Cuasiexperimental $(n=72)$
$0,17(0,16)$
$0,15(0,20)$

\section{Doble rol (víctima-agresor)}

Ciberagresión
Control $(n=33)$
$0,58(0,37)$

\begin{tabular}{|c|c|c|c|}
\hline \multirow{2}{*}{ Ciberagresion } & & & \\
\hline & Cuasiexperimental $(n=62)$ & $0,63(0,46)$ & $0,28(0,40)$ \\
\hline \multirow{2}{*}{ Cibervictimización } & Control $(n=33)$ & $0,65(0,37)$ & $0,41(0,41)$ \\
\hline & Cuasiexperimental $(n=63)$ & $0,63(0,42)$ & $0,34(0,37)$ \\
\hline \multicolumn{4}{|c|}{ No implicados } \\
\hline \multirow{2}{*}{ Ciberagresión } & Control $(n=1.015)$ & $0,04(0,08)$ & $0,06(0,16)$ \\
\hline & Cuasiexperimental $(n=1.406)$ & $0,05(0,10)$ & $0,05(0,18)$ \\
\hline \multirow{2}{*}{ Cibervictimización } & Control $(n=1.016)$ & $0,07(0,11)$ & $0,09(0,20)$ \\
\hline & Cuasiexperimental $(n=1.397)$ & $0,08(0,13)$ & $0,09(0,20)$ \\
\hline
\end{tabular}
$0,40(0,36)$

Fuente: elaboración propia.

\section{Discusión}

El presente estudio tenía como objetivo conocer si es posible disminuir el cyberbullying mediante una intervención psicoeducativa implementada por el propio profesorado, sin la intervención de agentes externos, teniendo en cuenta las potenciales diferencias en función del género, el curso y los roles del alumnado implicado (víctima, agresor, doble rol o 
no implicados). Para ello, se diseñó una intervención psicoeducativa basada en la evidencia científica y se partió de la idea de que el profesorado es consciente de la influencia del cyberbullying en la convivencia escolar y en el bienestar del alumnado y, por ello, está interesado en formarse y adquirir un nivel de competencia profesional adecuado para actuar y prevenir este tipo de violencia online. No obstante, si no es así, la campaña de sensibilización incluida facilitaría esta predisposición. Así, se asumió que si el profesorado aumentaba su competencia a través del material educativo facilitado, se conseguiría disminuir el cyberbullying (Zych et al., 2015).

A pesar de que muchas intervenciones de este tipo suelen ser efectivas en la reducción de la victimización (Gaffney et al., 2019), suelen ser implementadas por expertos, tienen un coste elevado para los centros educativos y no suelen ser efectivas en la misma medida en la reducción de la agresión. Sin embargo, este trabajo ha mostrado que con escaso coste y sin requerir una gran especialización, es posible que los centros educativos realicen una intervención psicoeducativa efectiva en la disminución de ambas dimensiones del cyberbullying. Los resultados permiten constatar que la implicación en ciberagresión y cibervictimización se mantiene e, incluso, aumenta si no se desarrolla la intervención, mientras que si se lleva a cabo una intervención que siga las directrices aquí indicadas, disminuyen ambas dimensiones del cyberbullying. Se ha constatado que a medida que estos fenómenos se mantienen en el tiempo, aumenta el posible daño a todos los implicados (Livingstone y Smith, 2014), por lo que los resultados obtenidos son muy positivos para la mejora de la situación frente a este tipo de violencia. Estos resultados respaldan la idea de que es posible prevenir e intervenir ante riesgos de las TIC como el cyberbullying desde los propios centros educativos a través de un material eficaz y con pocos recursos.

Concretamente, se logra impactar de manera destacada en los chicos, puesto que disminuye tanto la ciberagresión como la cibervictimización. En el caso de las chicas, logra disminuir la ciberagresión. No obstante, también se aprecia una tendencia a disminuir la cibervictimización. En este sentido, dado que son las chicas las que sufren más ciberagresiones y son más victimizadas (Sanjuán, 2019; Ybarra et al., 2014), es necesario que se profundice en mayor medida en la atención a las diferencias de género y se incida en las formas específicas de victimización de las chicas (Dobson y Ringrose, 2016; Li, 2006).

También se ha apreciado una tendencia positiva a reducir el cyberbullying en todos los cursos de educación secundaria en los que se ha implementado la intervención, pero ha mostrado ser especialmente efectiva en la reducción de la cibervictimización

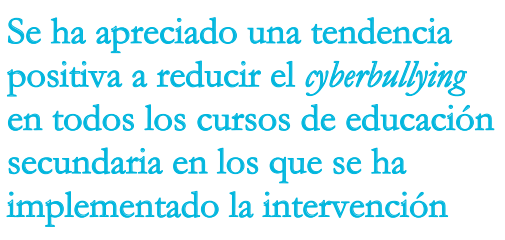

Se ha apreciado una tendencia positiva a reducir el cyberbullying en todos los cursos de educación secundaria en los que se ha implementado la intervención 
en $1 .^{\circ}$ de ESO y de la ciberagresión en $2 .^{\circ}$ y $3 .^{\circ}$ de ESO. En el caso de $4 .^{\circ}$ de ESO, se aprecia una disminución generalizada de este tipo de violencia en ambos grupos, tanto control como experimental. Este hecho es fundamental, puesto que, a diferencia del bullying (Tsitsika et al., 2014), el cyberbullying aumenta con la edad (UNESCO, 2019). Además, teniendo en cuenta que puede surgir por primera vez entre los 8 y los 9 años (Sanjuán, 2019), es primordial intervenir y ofrecer estrategias de manera temprana, adaptándose a la edad de los menores.

Asimismo, se obtuvieron resultados especialmente positivos en la mejora de la cibervictimización en víctimas y agresores. En el caso de la ciberagresión, aunque se aprecia una tendencia a disminuir, existe una mayor dificultad para modificar dicha conducta. Metaanálisis previos han constatado que este tipo de intervenciones pue-
En este estudio de investigación se obtuvieron resultados especialmente positivos en la mejora de la cibervictimización en víctimas y agresores den llegar a ser efectivas para disminuir la agresión cuando es especialmente alta, pero no para disminuir su potencial incremento (Ttofi y Farrington, 2011; Wilson y Lipsey, 2007). Es por ello por lo que es necesario realizar esfuerzos complementarios que aborden específicamente la agresión. Igualmente, el doble rol, en el que el alumnado es víctima y agresor al mismo tiempo, ha mostrado ser el que presenta mayores problemas de ajuste psicológico (Gámez-Guadix et al., 2015; Wachs, 2012), por lo que incidir en este tipo de implicación puede necesitar también un mayor esfuerzo educativo e, incluso, una intervención clínica. No obstante, la intervención sí ha mostrado ser útil, en este sentido, para el alumnado no implicado, puesto que ha logrado disminuir su implicación como agresores antes de que suceda. Además, se puede apreciar un aumento del número de alumnado no implicado en cyberbullying. Así, existe un mayor riesgo de convertirse en agresor o agresora en aquellos centros educativos donde no se ha aplicado la intervención. Esto puede deberse a la toma de consciencia y al aumento de un mayor criterio moral y empatía (Zych et al., 2015). Se evidencia un cambio de tendencia en la que la autorregulación, como elemento clave, ha podido actuar como inhibidor de la agresión (Vazsonyi et al., 2012).

Por tanto, los resultados avalan la posibilidad de desarrollar intervenciones psicoeducativas eficaces para la actuación ante el cyberbullying con un bajo coste para los centros educativos y sin depender de agentes externos. Además, la evaluación permite conocer las estrategias y orientaciones que han mostrado ser eficaces para ello. No es necesario que el profesorado tenga un dominio experto del entorno virtual para prevenir sus riesgos, sino que las estrategias que se han desarrollado durante mucho tiempo para prevenir otro tipo de violencias (Zych et al., 2019), como la autorregulación o partir de las ideas del alumnado, pueden
Los resultados avalan la posibilidad de desarrollar intervenciones psicoeducativas eficaces para la actuación ante el cyberbullying con un bajo coste para los centros educativos y sin depender de agentes externos. Además, la evaluación permite conocer las estrategias y orientaciones que han mostrado ser eficaces para ello 
transferirse también al contexto digital. Esto, unido al desarrollo de intervenciones de calidad, permite abordar de manera muy satisfactoria la prevención de este tipo de violencia online. Así, dada la demanda existente por parte de la comunidad científica de ofrecer recursos al profesorado que permitan afrontar el cyberbullying (Tangen y Campbell, 2010) y la evidencia de que el mantenimiento en el tiempo de este fenómeno intensifica su impacto (Livingstone y Smith, 2014), el desarrollo de intervenciones que sigan las pautas que aquí se presentan debe convertirse en una tarea imprescindible en el día a día de los centros educativos.

Por último, a pesar del avance que supone el presente estudio, es necesario tener en cuenta sus limitaciones. Concretamente, las principales limitaciones están relacionadas con el uso de una muestra incidental por accesibilidad y los instrumentos de autoinforme, que siempre conllevan el riesgo de obtener respuestas socialmente deseables o con poca precisión. Además, es necesario tener en cuenta que no se hizo un seguimiento extremadamente riguroso de la implementación de la intervención y en algunas clases no se realizaron alguna de las sesiones. No obstante, quizás, la limitación más relevante es la necesidad de mejorar aún más la intervención para que contemple una formación más específica que atienda las diferencias de género y los comportamientos de agresión. Las futuras líneas de investigación podrían abordar estas limitaciones.

\section{Referencias bibliográficas}

Álvarez-García, D., Barreiro-Collazo, A. y Núñez, J.-C. (2017). Cyberaggression among adolescents: prevalence and gender differences. Comunicar, 25(50), 89-97. https://doi. org/10.3916/C50-2017-08

Ausubel, D. P. (1963). The psychology of meaningful verbal learning. Grune \& Stratton. https://psycnet.apa.org/record/196410399-000

Bernete García, F. (2010). Usos de las TIC, relaciones sociales y cambios en la socialización de las y los jóvenes. Revista de Estudios de Juventud, 88, 97-114. https:// dialnet.unirioja.es/servlet/articulo?co digo $=3254537$

Bindesbøl Holm Johansen, K., Pedersen, B. M. y Tjørnhøj-Thomsen, T. (2018). Visual gossiping: non-consensual 'nude' sharing among young people in Denmark. Culture, Health \& Sexuality, 1-16. https://doi.org/1 0.1080/13691058.2018.1534140

Calvo Hernández, P. y Marrero Rodríguez, G. (2004). La convivencia en los centros escolares como factor de calidad. El Guiniguada. Revista de Investigaciones y Experiencias en Ciencias de la Educación, 13. https://ojsspdc.ulpgc.es/ojs/index.php/ EIGuiniguada/article/view/581

Casas, J. A., Ortega-Ruiz, R. y Del Rey, R. (2015). Bullying: the impact of teacher management and trait emotional intelligence. British Journal of Educational Psychology, 85(3), 407-423. https://doi.org/10.1111/bjep. 12082

Cerezo Ramírez, F. y Rubio Hernández, F. J. (2017). Medidas relativas al acoso escolar 
y ciberacoso en la normativa autonómica española. Un estudio comparativo. Revista Electrónica Interuniversitaria de Formación del Profesorado, 20(1), 113-126. https:// doi.org/10.6018/REIFOP/20.1.253391

Del Rey, R., Casas, J. A. y Ortega-Ruiz, R. (2017). Desarrollo y validación de la escala de convivencia escolar (ECE). Universitas Psychologica, 16(1), 1-11. https://doi. org/10.11144/Javeriana.upsy16-1.dvec

Del Rey, R., Casas, J. A., Ortega-Ruiz, R., Schultze-Krumbholz, A., Scheithauer, H., Smith, P., Thompson, F., Barkoukis, V., Tsorbatzoudis, H., Brighi, A., Guarini, A., Pyżalski, J. y Plichta, P. (2015). Structural validation and cross-cultural robustness of the European cyberbullying intervention project questionnaire. Computers in Human Behavior, 50, 141-147. https://doi.org/10.1016/j.chb. 2015.03.065

Del Rey, R., Mora-Merchán, J. A., Casas, J. A., Ortega-Ruiz, R. y Elipe, P. (2018). «Asegúrate» Program: effects on cyberaggression and its risk factors. Comunicar, 26(56), 39-48. https://doi.org/10.3916/C5 6-2018-04

Del Rey, R., Ortega-Ruiz, R. y Casas, J. A. (2019). Asegúrate: an intervention program against cyberbullying based on teachers' commitment and on design of its instructional materials. International Journal of Environmental Research and Public Health, 16(3), 434. https://doi.org/10.3390/ijerph1 6030434

Delors, J. (1996). La educación encierra un tesoro. Informe a la UNESCO de la Comisión Internacional sobre la Educación para el Siglo XXI. https://unesdoc.unesco.org/ ark:/48223/pf0000109590_spa

Dobson, A. S. y Ringrose, J. (2016). Sext education: pedagogies of sex, gender and shame in the schoolyards of tagged and exposed. Sex Education, 16(1), 8-21. https://doi.org/ 10.1080/14681811.2015.1050486

Espelage, D. L. y Swearer, S. M. (2008). Current perspectives on linking school bullying research to effective prevention strategies. En T. W. Miller (Ed.), School Violence and Primary Prevention (pp. 335-353). Springer. https://doi.org/10.1007/978-0-3 87-77119-9_17

Fahy, A. E., Stansfeld, S. A., Smuk, M., Smith, N. R., Cummins, S. y Clark, C. (2016). Longitudinal associations between cyberbullying involvement and adolescent mental health. Journal of Adolescent Health, 59(5), 502-509. https://doi.org/10.1016/j.jadohe alth.2016.06.006

Fernández-Montalvo, J., Peñalva Vélez, A. e Irazabal, I. (2015). Internet use habits and risk behaviours in preadolescence. Comunicar, 22(44), 113-121. https://doi.org/10. 3916/C44-2015-12

Frankel, A. S., Bass, S. B., Patterson, F., Dai, T. y Brown, D. (2018). Sexting, risk behavior, and mental health in adolescents: an examination of 2015 Pennsylvania youth risk behavior survey data. Journal of School Health, 88(3), 190-199. https://doi.org/10. 1111/josh.12596

Gaffney, H., Farrington, D. P., Espelage, D. L. y Ttofi, M. M. (2019). Are cyberbullying intervention and prevention programs effective? A systematic and meta-analytical review. Aggression and Violent Behavior, 45, 134-153. https://doi.org/10.1016/j.avb.20 18.07.002

Gámez-Guadix, M., Gini, G. y Calvete, E. (2015). Stability of cyberbullying victimization among adolescents: prevalence and association with bully-victim status and psychosocial adjustment. Computers in 
Human Behavior, 53, 140-148. https://doi. org/10.1016/j.chb.2015.07.007

Garaigordobil, M. (2015). Ciberbullying en adolescentes y jóvenes del País Vasco: cambios con la edad. Anales de Psicología, 31(3), 1.069-1.076. https://doi.org/10.6018/ analesps.31.3.179151

Garaigordobil, M. y Martínez-Valderrey, V. (2015). Effects of cyberprogram 2.0 on faceto-face bullying, cyberbullying, and empathy. Psicothema, 27(1), 45-51. https://doi. org/10.7334/psicothema2014.78

Garmendia Larrañaga, M., Jiménez Iglesias, E., Casado, M. Á. y Mascheroni, G. (2016). Net Children Go Mobile: riesgos y oportunidades en internet y el uso de dispositivos móviles entre menores españoles (20102015). https://bit.ly/2viYYc2

Hinduja, S. y Patchin, J. W. (2008). Cyberbullying: an exploratory analysis of factors related to offending and victimization. Deviant Behavior, 29(2), 129-156. https:// doi.org/10.1080/01639620701457816

Kowalski, R. M., Giumetti, G. W., Schroeder, A. N. y Lattanner, M. R. (2014). Bullying in the digital age: a critical review and meta-analysis of cyberbullying research among youth. Psychological Bulletin, 140(4), 1.073-1.137. https://doi.org/10.1037/a0035618

Lenhart, A., Smith, A. y Anderson, M. (2015). Teens, Technology and Romantic Relationships: From Flirting to Breaking up, Social Media and Mobile Phones Are Woven into Teens' Romantic Lives. https://pewrsr.ch/2 MI51IX

Li, Q. (2006). Cyberbullying in schools. School Psychology International, 27(2), 157-170. https://doi.org/10.1177/0143034306064547

Livingstone, S. (2014). Developing social media literacy: how children learn to interpret risky opportunities on social network sites. Communications, 39(3), 283-303. https://doi.org/ 10.1515/commun-2014-0113

Livingstone, S. y Smith, P. (2014). Annual research review. Harms experienced by child users of online and mobile technologies: the nature, prevalence and management of sexual and aggressive risks in the digital age. Journal of Child Psychology and Psychiatry, 55(6), 635-654. https://doi.org/ 10.1111/jcpp.12197

López-Pradas, I. C., Romera, E. M., Casas, J. A. y Ortega-Ruiz, R. (2017). El cibercotilleo y el ciberacoso en la enseñanza primaria. Psicología Educativa, 23(2), 73-80. https:// doi.org/10.1016/j.pse.2017.05.007

Menesini, E., Nocentini, A., Palladino, B. E., Frisén, A., Berne, S., Ortega-Ruiz, R., Calmaestra, J., Scheithauer, H., SchultzeKrumbholz, A., Luik, P., Naruskov, K., Blaya, C., Berthaud, J. y Smith, P. K. (2012). Cyberbullying definition among adolescents: a comparison across six European countries. Cyberpsychology, Behavior, and Social Networking, 15(9), 455-463. https:// doi.org/10.1089/cyber.2012.0040

Mitchell, K. J., Finkelhor, D., Jones, L. M. y Wolak, J. (2012). Prevalence and characteristics of youth sexting: a national study. Pediatrics, 129(1), 13-20. https://doi.org/ 10.1542/peds.2011-1730

Nacimiento Rodríguez, L., Rosa Pantoja, I. y Mora-Merchán, J. A. (2017). Valor predictivo de las habilidades metacognitivas en el afrontamiento en situaciones de bullying y cyberbullying. Informes Psicológicos, 17(2), 135-158. https://doi.org/10.18566/infpsic. v17n2a08

Ortega-Ruiz, R., Casas, J. A. y Del Rey, R. (2014). Towards the construct of cyberconvivencia. Journal for the Study of Education 
and Development. Infancia y Aprendizaje, 37, 602-628. https://doi.org/10.1080/02103 702.2014 .957537

Ortega-Ruiz, R., Del Rey, R. y Casas, J. A. (2016). Evaluar el bullying y el cyberbullying validación española del EBIP-Q y del ECIP-Q. Psicología Educativa, 22(1), 71-79. https:// doi.org/10.1016/j.pse.2016.01.004

Ortega Ruiz, R., Del Rey, R. y Sánchez, V. (2012). Nuevas dimensiones de la convivencia escolar juvenil. Ciberconducta y relaciones en la red: ciberconvivencia. https://sede. educacion.gob.es/publiventa/d/15394/19/0

Patchin, J. W. e Hinduja, S. (2012). Cyberbullying Prevention and Response: Expert Perspectives. Routledge.

Powell, K. C. y Cody, K. J. (2009). Cognitive and social constructivism: developing tools for and effective classroom. Education, 130(2), 241-250.

Rachoene, M. y Oyedemi, T. (2015). From selfexpression to social aggression: cyberbullying culture among South African youth on Facebook. Communicatio, 41(3), 302319. https://doi.org/10.1080/02500167.2 015.1093325

Rimal, R. N., Lapinski, M. K., Cook, R. J. y Real, K. (2005). Moving toward a theory of normative influences: how perceived benefits and similarity moderate the impact of descriptive norms on behaviors. Journal of Health Communication, 10(5), 433-450. https://doi. org/10.1080/10810730591009880

Rodríguez-Gómez, D., Castro, D. y Meneses, J. (2018). Usos problemáticos de las TIC entre jóvenes en su vida personal y escolar. Comunicar, 27(56), 91-100. https://doi.org/ 10.3916/C56-2018-09

Romera, E. M., Herrera-López, M., Casas, J. A., Ortega-Ruiz, R. y Del Rey, R. (2018). How much do adolescents cybergossip? Scale development and validation in Spain and Colombia. Frontiers in Psychology, 9, 126. https://doi.org/10.3389/fpsyg.2018.00126

Rubio Hernández, F. J., Díaz López, A. y Cerezo Ramírez, F. (2019). Bullying y cyberbullying: la respuesta de las comunidades autónomas. Revista Electrónica Interuniversitaria de Formación del Profesorado, 22(1). https://doi.org/10.6018/reifop.22.1.332311

Sanjuán, C. (2019). Violencia viral: análisis de la violencia contra la infancia y la adolescencia en el entorno digital. Save the Children. https://www.savethechildren.es/publicacio nes/informe-violencia-viral-y-online-contrala-infancia-y-la-adolescencia

Sastre, A., Calmaestra, J., Escorial, A., García, P., Del Moral, C., Perazzo, C. y Ubrich, T. (2016). Yo a eso no juego. Bullying y cyberbulllying en la infancia. Save the Children. https://www.savethechildren.es/sites/de fault/files/imce/docs/yo_a_eso_no_juego.pdf

Seiler, S. J. y Navarro, J. N. (2014). Bullying on the pixel playground: investigating risk factors of cyberbullying at the intersection of children's online-offline social lives. Cyberpsychology. Journal of Psychosocial Research on Cyberspace, 8(4). https://doi.org/ 10.5817/CP2014-4-6

Slonje, R., Smith, P. K. y Frisén, A. (2013). The nature of cyberbullying, and strategies for prevention. Computers in Human Behavior, 29(1), 26-32. https://doi.org/10.1016/J.CH B.2012.05.024

Tangen, D. y Campbell, M. (2010). Cyberbullying prevention: one primary schools approach. Australian Journal of Guidance and Counselling, 20(2), 225-234. https://doi.org/10.1375/ ajgc.20.2.225

Tokunaga, R. S. (2010). Following you home from school: a critical review and synthesis of research on cyberbullying victimization. 
Computers in Human Behavior, 26(3), $277-$ 287. https://doi.org/10.1016/j.chb.2009. 11.014

Tsitsika, A. K., Barlou, E., Andrie, E., Dimitropoulou, C., Tzavela, E. C., Janikian, M. y Tsolia, M. (2014). Bullying behaviors in children and adolescents: "An ongoing story". Frontiers in Public Health, 2, 7. https://doi. org/10.3389/fpubh.2014.00007

Ttofi, M. M. y Farrington, D. P. (2011). Effectiveness of school-based programs to reduce bullying: a systematic and meta-analytic review. Journal of Experimental Criminology, 7(1), 27-56. https://doi.org/10.1007/ s11292-010-9109-1

Turkle, S. (2008). Always-on/Always-on-you: the tethered self. En J. E. Katz (Ed.), Handbook of Mobile Communication Studies (pp. 121-137). MIT Press Scholarship Online.

UNESCO. (2019). Behind the numbers: ending school violence and bullying. https://unes doc.unesco.org/ark:/48223/pf0000366483

Vazsonyi, A. T., Machackova, H., Sevcikova, A., Smahel, D. y Cerna, A. (2012). Cyberbullying in context: direct and indirect effects by low self-control across 25 European countries. European Journal of Developmental Psychology, 9(2), 210-227. https:// doi.org/10.1080/17405629.2011.644919

Villacampa, C. (2017). Teen sexting: prevalence, characteristics and legal treatment. International Journal of Law, Crime and Justice, 49, 10-21. https://doi.org/10.1016/j. ijlcj.2017.01.002
Waasdorp, T. E. y Bradshaw, C. P. (2015). The overlap between cyberbullying and traditional bullying. Journal of Adolescent Health, 56(5), 483-488. https://doi.org/10.1016/j. jadohealth.2014.12.002

Wachs, S. (2012). Moral disengagement and emotional and social difficulties in bullying and cyberbullying: differences by participant role. Emotional and Behavioural Difficulties, 17(3-4), 347-360. https://doi.org/10.1080/ 13632752.2012 .704318

Wilson, S. J. y Lipsey, M. W. (2007). Schoolbased interventions for aggressive and disruptive behavior. Update of a meta-analysis. American Journal of Preventive Medicine, 33(2 suplemento), S130-S143. https://doi. org/10.1016/j.amepre.2007.04.011

Ybarra, M. L., Espelage, D. L. y Mitchell, K. J. (2014). Differentiating youth who are bullied from other victims of peer-aggression: the importance of differential power and repetition. Journal of Adolescent Health, 55(2), 293-300. https://doi.org/10.1016/j.jadoheal th.2014.02.009

Zych, I., Farrington, D. P. y Ttofi, M. M. (2019). Bullying and cyberbullying: protective factors and effective interventions. Aggression and Violent Behavior, 45, 1-3. https://doi. org/10.1016/j.avb.2018.08.006

Zych, I., Ortega-Ruiz, R. y Del Rey, R. (2015). Systematic review of theoretical studies on bullying and cyberbullying: Facts, knowledge, prevention, and intervention. Aggression and Violent Behavior, 23, 1-21. https://doi.org/ 10.1016/j.avb.2015.10.001 